\title{
Acral lentiginous melanoma in the Turkish population and a new dermoscopic clue for the diagnosis
}

\author{
Fezal Ozdemir ${ }^{1}$, Micol A. Errico ${ }^{2}$, Banu Yaman $^{3}$, Isil Karaarslan ${ }^{1}$
}

\author{
1 Ege University, Medical Faculty, Dermato-Oncology Unit, Department of Dermatology, Izmir, Turkey \\ 2 Department of Medical Sciences, University of Turin, Turin, Italy \\ 3 Ege University, Medical Faculty, Department of Pathology, Izmir, Turkey
}

\begin{abstract}
Key words: acral lentiginous melanoma, amelanotic melanoma, nail unit melanoma, parallel-ridge pattern, dermoscopy
Citation: Ozdemir F, Errico MA, Yaman B, Karaarslan I. Acral lentiginous melanoma in the Turkish population and a new dermoscopic clue for the diagnosis. Dermatol Pract Concept. 2018;8(2)140-148. DOI: https://doi.org/10.5826/dpc.0802a14
\end{abstract}

Received: July 31, 2017; Accepted: February 7, 2018; Published: April 30, 2018

Copyright: $\odot 2018$ Ozdemir et al. This is an open-access article distributed under the terms of the Creative Commons Attribution License, which permits unrestricted use, distribution, and reproduction in any medium, provided the original author and source are credited.

Funding: None.

Competing interests: The authors have no conflicts of interest to disclose.

All authors have contributed significantly to this publication.

Corresponding author: Micol Alessandra Errico, MD, Dermatology Unit, Department of Medical Sciences, University of Turin, Turin, Italy. Tel. +390165545426; Fax. +390165545583. Email: micolerr@libero.it

ABSTRACT Background: The incidence of acral lentiginous melanoma (ALM) in the white population is low. Dermoscopy enhances diagnosis of ALM; however, diagnostic accuracy may sometimes be poor due to the considerable proportion of amelanotic ALM variants.

Objectives: To calculate the proportion of ALM among all melanoma subtypes and to determine the frequency of dermoscopic features of ALM in the Turkish population.

Methods: Out of 612 melanomas, there were 70 cases of ALM, of which 46 showed sufficient image quality for retrospective study of dermoscopic features. Data from patients and their lesions was classified according to clinical features and histopathologic parameters. The dermoscopic variables evaluated were based on pertinent literature on dermoscopy of acral melanocytic neoplasms.

Results: The prevalence of ALM among all melanoma subtypes was $11.4 \%$. Parallel-ridge pattern (PRP) was detected in $60.8 \%$ of cases and irregular diffuse pigmentation (IDP) in $28.3 \%$. The ALMs were amelanotic in $24 \%$, showing an atypical vascular pattern in all cases; a new dermoscopic pattern, named "vascularized parallel-ridge pattern" (VPRP), was detected in $13 \%$ of ALMs. Irregular lines were observed in $81.8 \%$ of subungual melanomas and were often associated with a multicolored background.

Conclusions: ALM has site-specific dermoscopic patterns, with PRP being the most prevalent pattern. The newly described VPRP pattern may be an additional clue for ALM diagnosis, especially in thin amelanotic melanomas. 


\section{Background}

Acral lentiginous melanoma (ALM) accounts for 2-8\% of all melanomas in Caucasians [1-3], $15 \%$ to $35 \%$ in darkskinned [4-7], and up to $60 \%$ in Asians [8]. The nail unit or subungual melanoma, an anatomical variant of ALM, is even less frequent, accounting for 1-2\% of all melanomas in white population [9-12] and 10-20\% in Asians [13,14]. "Lentiginous" relates to a histopathologic pattern of proliferation of melanocytes along the dermoepidermal junction (DEJ) as single cells and small nests with areas of confluent growth, although not all acral melanomas display this specific pattern [15]. Of acral melanomas, ALM is the most prevalent histopathologic subtype (40\%-80\%) [16-18].

Due to the low incidence of ALM, clinical experience is limited to specialized centers. Moreover, the atypical clinical presentation and relatively high frequency of amelanotic variants are seen among ALMs. As a result, delayed diagnosis or misdiagnosis may lead to detection of ALM at a more advanced stage with poor prognosis [15].

Dermoscopy increases the diagnostic accuracy for early ALM diagnosis, helping to differentiate it from acral nevi [19-23]. ALM is typified by pigmentation along the skin ridges, termed "parallel-ridge pattern" (PRP), while acral nevi display mostly the "parallel-furrow pattern" (PFP) [2428]. Among ALMs, other dermoscopic patterns include the irregular diffuse pigmentation (IDP), defined as structureless, diffuse pigmentation with variable shades of brown to black color and without parallel disposition of pigment [22-24,28]; the multicomponent pattern, defined as exhibiting more than one predominant dermoscopic criteria [29] or a combination of pigmentation patterns [30]; and the polymorphic vascular pattern that is especially seen among amelanotic acral melanomas.

The aim of the present study was to calculate the proportion of ALM among all melanoma subtypes and to determine the frequency of dermoscopic features of ALM in the Turkish population.

\section{Methods}

All melanoma cases between 2005 and 2014 were identified retrospectively from the archive of the Dermato-Oncology Unit, Department of Dermatology of Ege University, Izmir, Turkey. Our institution did not require an Ethics Committee approval for this retrospective study of dermoscopic images. For all cases included in the study, the diagnosis of ALM was based on the histopathologic report. Images of all cases of ALM were reviewed; we excluded cases that lacked a dermoscopic image of the primary lesion or when image quality was insufficient for evaluation of pattern.
Dermoscopic pictures were taken prior to biopsy or excision using a polarized light immersion dermoscopic camera (DermLite Foto System, 3Gen, San Juan Capistrano, CA, USA) with oil immersion. In selected cases, we obtained additional images of normal appearing skin around the lesions or at the contralateral acral site.

The clinical and dermoscopic images of all included ALM cases in were analyzed independently by two authors (FO and IK). Clinical data included age, gender, and anatomic distribution. Histopathologic parameters included Breslow tumor thickness and Clark's level of invasion. The dermoscopic variables evaluated were based on pertinent literature on dermoscopy of acral melanocytic neoplasms [22,23,25$28,30,31]$. In nail apparatus melanomas, we also analyzed the pattern of involved periungual skin.

\section{Results}

There were 612 cases of melanoma in our database. Of these, $70(11.4 \%)$ were diagnosed as ALM. The patients' mean age was 61.2 (range 16-88), and 38 (54.3\%) were females. Eighty percent $(n=56)$ of melanomas were located on the feet (50 on plantar surfaces and 6 in toenails) and $20 \%$ on hands (5 on palmar surfaces and 9 in fingernails). The mean Breslow thickness was $3.84 \mathrm{~mm} ; 14.3 \%(\mathrm{n}=10)$ were classified as in situ melanomas, $11.4 \%(\mathrm{n}=8)$ were $<1 \mathrm{~mm}$, while $74.3 \%$ were thick melanomas (Breslow thickness $1-2 \mathrm{~mm}$ in 7 patients; $>2-4 \mathrm{~mm}$ in 23 patients; $>4 \mathrm{~mm}$ in 22 patients). The majority of melanomas $(47.1 \% ; n=33)$ exhibited Clark's level IV.

Of acral melanomas, we analyzed the dermoscopic features in 46 melanomas with sufficient image quality (Table 1). Among global features, PRP was seen in $60.8 \%$, IDP was seen in $28.3 \%$, and multicomponent pattern in $47.8 \%$. Among focal dermoscopic patterns, most frequent were the irregular fibrillar pattern (IFP) in $34.8 \%$ and PFP in $30.4 \%$. Among dermoscopic findings described in melanoma on nonglabrous skin, an abrupt edge was seen in $34.8 \%$, irregular streaks in $32.6 \%$, and blue-whitish veil (BWV) in $30.4 \%$. Most lesions (76.1\%) showed multiple colors, 52.2\% exhibited atypical vessels, and $39.1 \%$ showed ulceration.

We observed a new dermoscopic feature in $13.0 \% \quad(n=6)$ of the melanomas, termed henceforth as "vascularized parallel-ridge pattern" (VPRP). This new dermoscopic finding is defined as "erythema and dotted vessels filling the ridges and sparing the sulci" (Figures 1-5).

Of ALMs, 24\% were amelanotic melanomas, including 4 non-pigmented melanomas and 7 hypopigmented with some remnants of pigmentation under dermoscopy. Atypical vessels were observed in all lesions, with dotted vessels being most common (72.7\%, Figure 6). Of amelanotic melanomas, 4/11 (36.4\%) showed the VPRP pattern (Figures 1-3). 
TABLE 1. Dermoscopic patterns found in our 46 cases of acral lentiginous melanomas (ALM), including involvement of periungual skin in nail apparatus melanoma.

\begin{tabular}{|l|c|c|}
\hline Dermoscopic Patterns & $\begin{array}{c}\text { Number } \\
\text { of ALM } \\
\text { (n, total= 46) }\end{array}$ & $\begin{array}{c}\text { Percentage } \\
\text { of ALM } \\
\text { (\%) }\end{array}$ \\
\hline $\begin{array}{l}\text { Parallel-ridge pattern } \\
\text { (PRP) }\end{array}$ & 28 & 60.8 \\
\hline $\begin{array}{l}\text { Irregular diffuse } \\
\text { pigmentation (IDP) }\end{array}$ & 13 & 28.3 \\
\hline Other patterns: & \multicolumn{2}{|l|}{} \\
\hline Multicomponent & 22 & 47.8 \\
\hline Polymorphic vascular & 5 & 10.9 \\
\hline Starburst & 1 & 2.2 \\
\hline Parallel-furrow (focal) & 14 & 30.4 \\
\hline $\begin{array}{l}\text { Irregular fibrillar } \\
\text { (focal) }\end{array}$ & 16 & 34.8 \\
\hline Lattice-like (focal) & 1 & 2.2 \\
\hline Reticular (focal) & 2 & 4.3 \\
\hline Homogeneous (focal) & 1 & 2.2 \\
\hline Non-typical & 0 & 0 \\
\hline Negative fibrillar & 5 & 10.9 \\
\hline Abrupt edge & 16 & 34.8 \\
\hline $\begin{array}{l}\text { Irregular dots and } \\
\text { globules }\end{array}$ & 13 & 28.3 \\
\hline Irregular streaks & 15 & 32.6 \\
\hline Homogenous area & 11 & 23.9 \\
\hline Irregular blotch & 6 & 13 \\
\hline Blue-white veil & 14 & 30.4 \\
\hline Scar-like depigmentation & 11 & 23.9 \\
\hline Peppering or grey dots & 3 & 6.5 \\
\hline Ulceration & 18 & 39.1 \\
\hline Atypical vessels & 24 & 52.2 \\
\hline Multiple colors & 35 & 76.1 \\
\hline Vascularized PRP & 6 & 13 \\
\hline
\end{tabular}

Of nail unit melanomas, 11 of 15 cases $(73.3 \%)$ showed adequate dermoscopic image quality for evaluation. Hutchinson's sign (i.e., pigmentation of the periungual skin) was detected in all cases. The mean Breslow thickness was $1.73 \mathrm{~mm}$ and 2 cases were in situ (18.2\%). Melanonychia striata longitudinalis (MSL), i.e. a pigmented band of the nail plate, was observed in 9 cases $(81.8 \%)$; however, the bands were comprised of irregular lines with respect to their color, thickness, spacing and parallelism (Figures 7-8). Nail plate dystrophy was seen in 7 cases (64\%, Figure 9). The most prevalent dermoscopic feature corresponded to irregular lines $(81.8 \%)$ and multicolored background $(72.7 \%)$ (Table 2).

\section{Discussion}

In our series, the prevalence of ALM among the melanomas was $11.4 \%$, in contrast to the reported prevalence among Caucasians of 2-8\% [1-3]; this slightly higher prevalence may be related to Turkish ethnicity.

The most prevalent dermoscopic pattern in our series was the PRP, seen in $60.8 \%$ of ALMs, and in line with previous studies. Saida et al, who first described PRP [25], found this pattern in $86 \%$ of cases, with sensitivity and specificity of PRP for ALM diagnosis being $86.4 \%$ and $99 \%$, respectively [23]. Likewise, Thomas et al [31] and Argenziano et al [29] have reported that PRP was detectable in $53 \%$ and $65.3 \%$ of their cases. IDP has been reported as the second most common dermoscopic pattern of ALM; it is more suggestive of invasive melanoma [19,21,23,31]. In our study, IDP was found in $28.3 \%$. The prevalence of IDP reported in the literature is variable: Argenziano et al reported 13.6\% [29], Braun et al reported 20.5\% [30], Thomas et al 60\% [31] and Saida et al $85 \%$ [23]. We speculate that IDP is seen in lower frequency in thicker melanomas, as notably $60 \%$ of ALMs in our series reached Clark's level IV and V. With regard to other acral patterns, a benign pattern such as PFP can be observed at times [30] but only focally and always together with melanomaspecific criteria. Multicomponent pattern $(47.8 \%)$ and IFP $(34.8 \%)$ were the other most prevalent patterns, as expected, in the literature [19]. Additional melanoma-specific criteria, seen in melanomas on non-glabrous skin [32,33]—including abrupt edge, irregular dots and globules $(\mathrm{D} / \mathrm{G})$, streaks, and blue-whitish veil (BWV) - were observed in one-third of our series; in addition, the high frequency of ulceration $(40 \%)$, atypical vessels $(>50 \%)$, and notably multiple colors $(76 \%)$ are again attributable to the high proportion of thick melanomas in our series [19].

About one-fifth of cases were nail unit melanomas mainly exhibiting an irregular MSL pattern, with variability in lines, color, thickness, spacing and parallelism, in addition to Hutchinson's sign seen in all cases. Notably, the usual finding of brown background, as previously reported by Thomas et al [31], was replaced by a multicolored background in most of our cases $(73 \%)$; we find this to be a useful diagnostic clue.

About one-quarter were amelanotic melanomas, in line with the previous reported frequencies by Thomas et al (28\%$34 \%)$ [31], and Kato and coworkers (19.6\%) in the Japanese population [34]. Furthermore, all melanomas exhibited atypical vessels, and in fact, the lack or scarcity of pigmentation allowed a clear visualization of the dermoscopic vascular pattern. Argenziano et al found that dotted vessels were detectable in $22.7 \%$ of melanomas, while erythema (defined as pinkish color within areas of regression or at the border of the lesion) was combined with different types of vessels in 43/159 melanomas [35]. Erythema was also slightly more prevalent among in situ melanomas, compared with invasive 


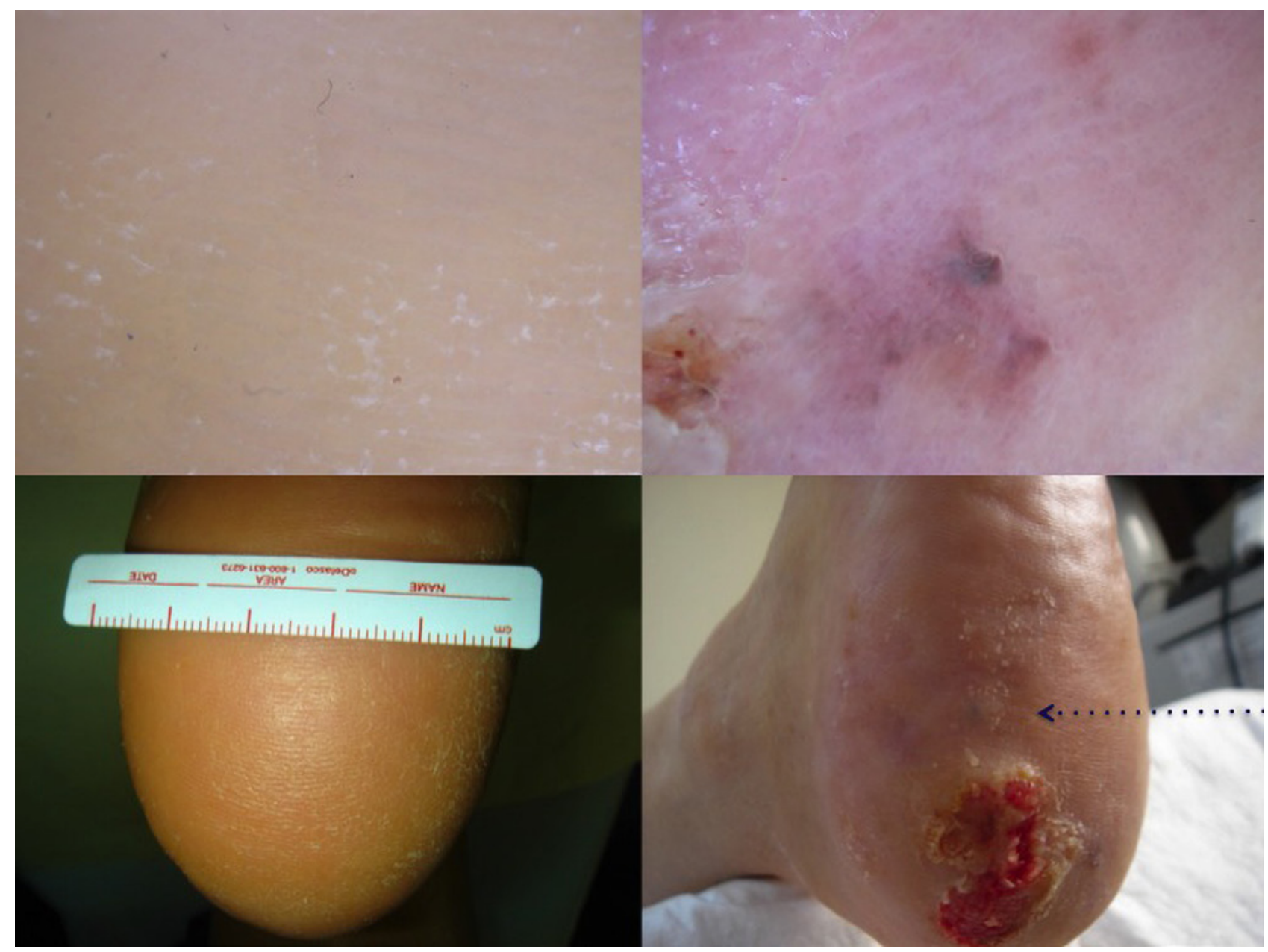

Figure 1. Vascularized parallel-ridge pattern (VPRP). Acral lentiginous melanoma, Breslow $3.3 \mathrm{~mm}$, Clark III. At dermoscopic evaluation, presence of erythema and some dotted vessels on the ridges, sparing the sulci (right). No erythema is detectable on the other healthy sole (left). [Copyright: (2018 Ozdemir et al.]

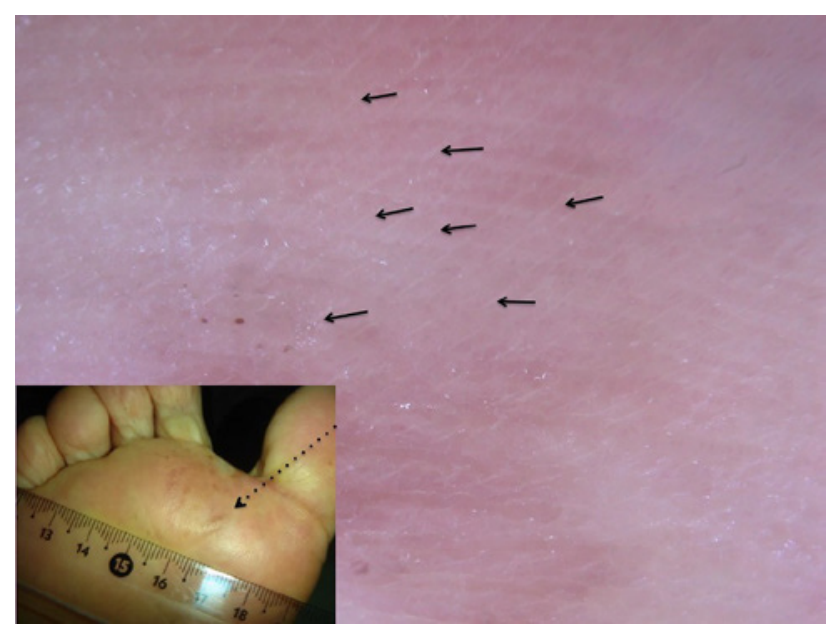

Figure 2. Vascularized parallel-ridge pattern (VPRP). Acral lentiginous melanoma, Breslow $0.8 \mathrm{~mm}$, Clark II. Evidence of erythema and few dotted vessels on the ridges, together with negative fibrillary pattern; white lines corresponding to eccrine ducts in the horny layer are seen throughout the lesion (arrows). [Copyright: (C2018 Ozdemir et al.]

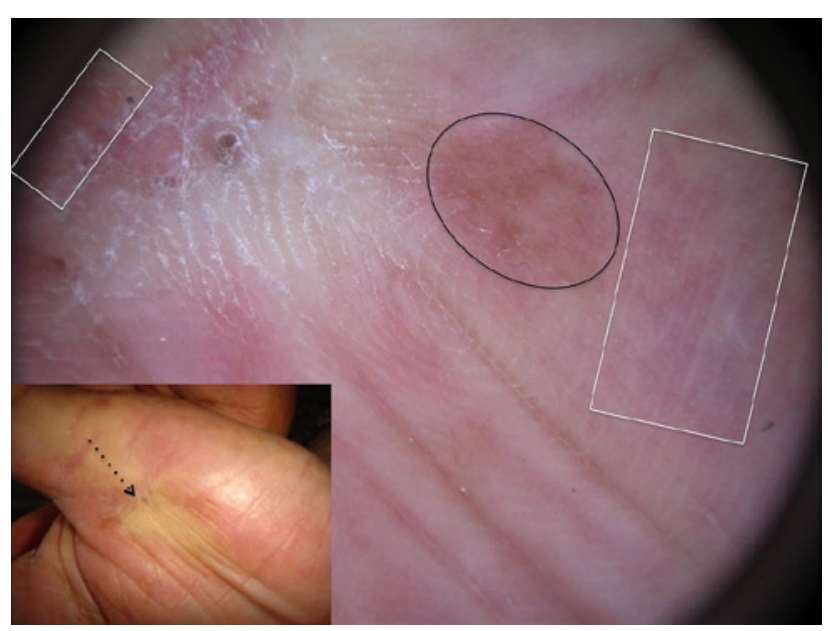

Figure 3. Parallel-ridge pattern (PRP) and vascularized PRP (VPRP). Recurrent, in situ, acral lentiginous melanoma (original lesion was ALM, Breslow $2.5 \mathrm{~mm}$, Clark IV). Both patterns are detectable sideby-side (PRP in circle, VPRP in rectangles). [Copyright: $\odot 2018$ Ozdemir et al.] 


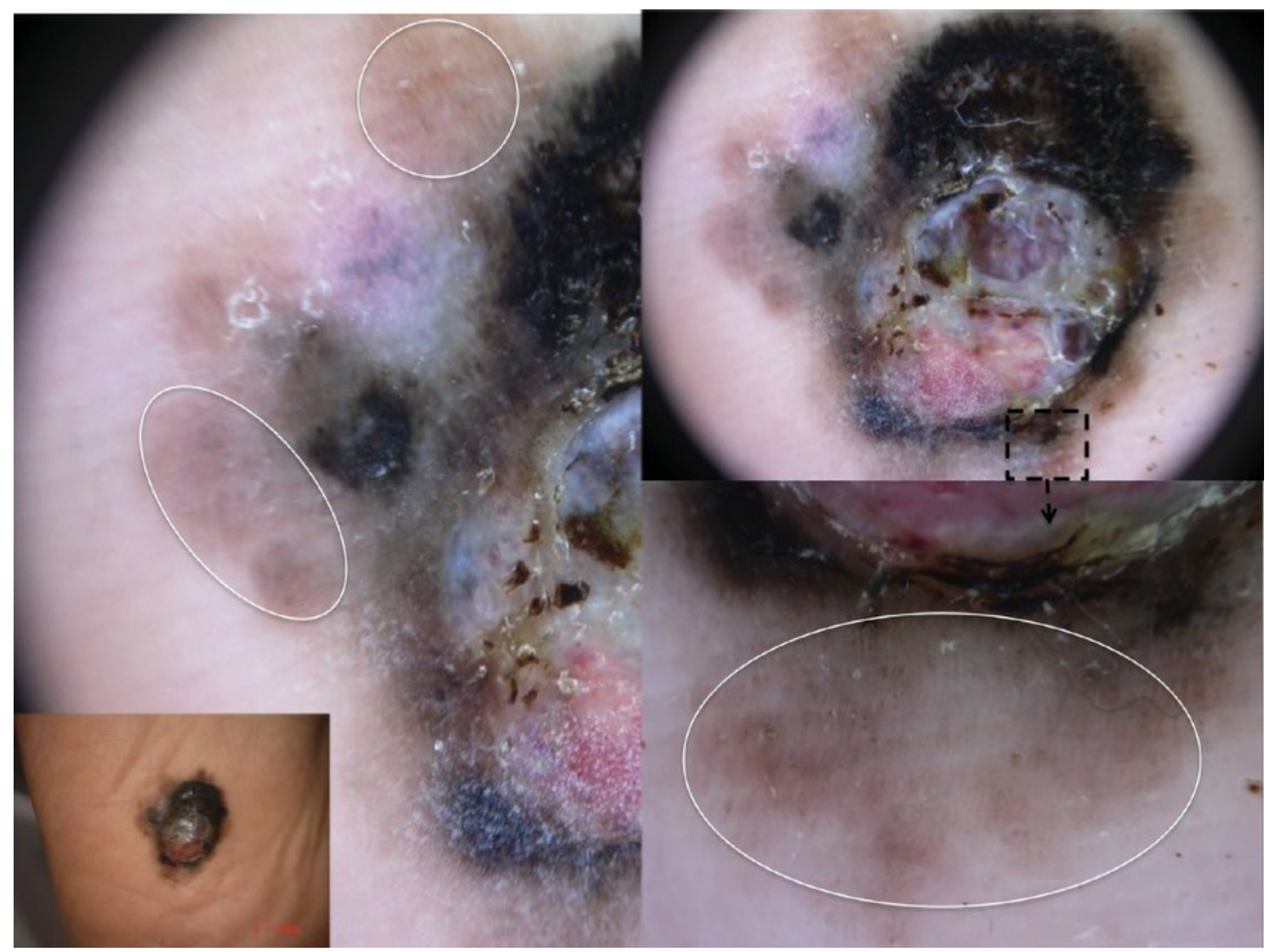

Figure 4. Vascularized PRP (VPRP). Acral lentiginous melanoma, Breslow $4.39 \mathrm{~mm}$, Clark IV. VPRP (in circles) is detectable along with remnants of pigmentation and gray dots. [Copyright: @2018 Ozdemir et al.]

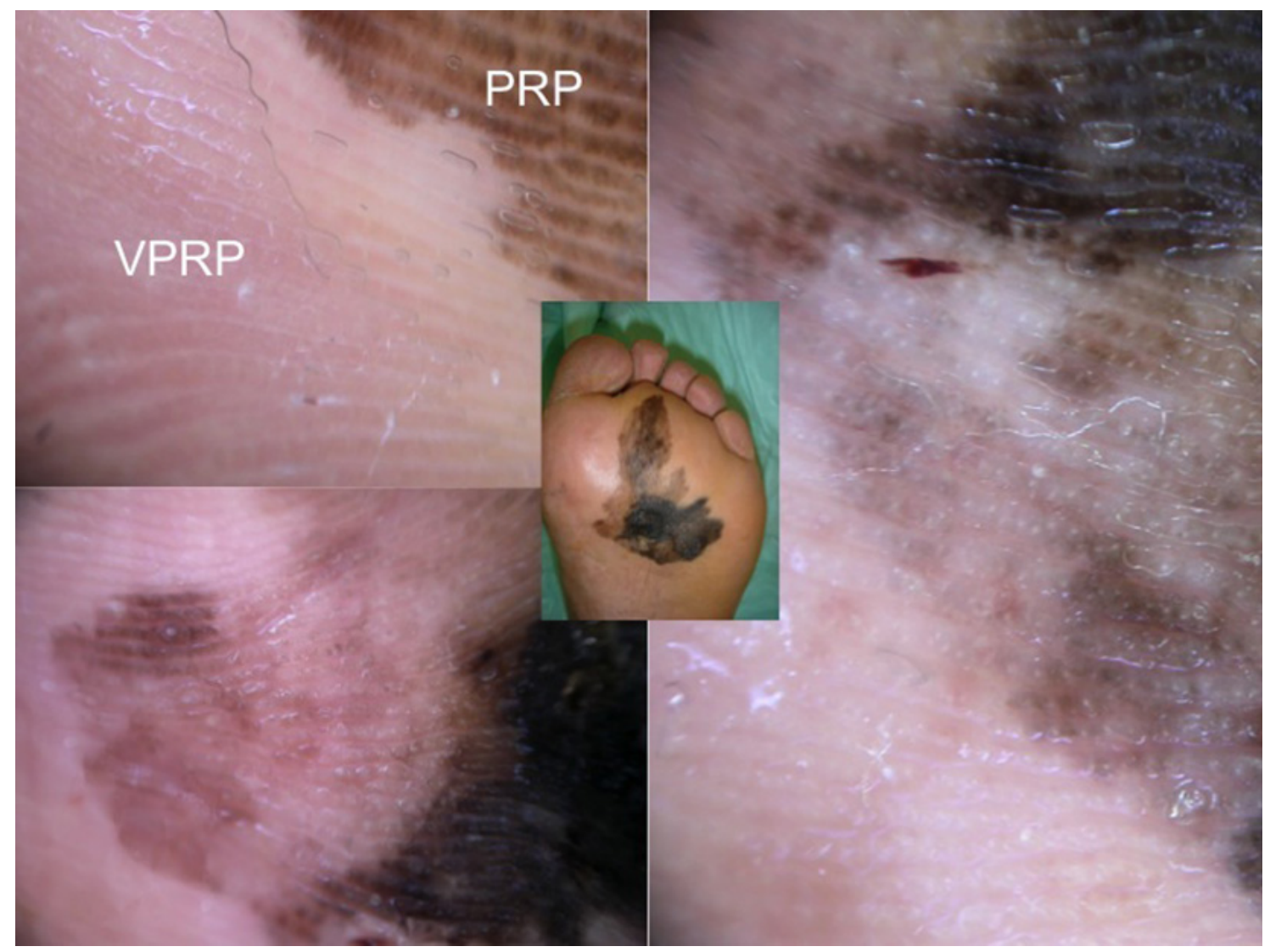

Figure 5. Parallel-ridge pattern (PRP) and vascularized PRP (VPRP). Acral lentiginous melanoma, Breslow $2.26 \mathrm{~mm}$, Clark IV. Both patterns are seen side-by-side (top left) and intermingled with each other (bottom left and right). [Copyright: C2018 Ozdemir et al.] 


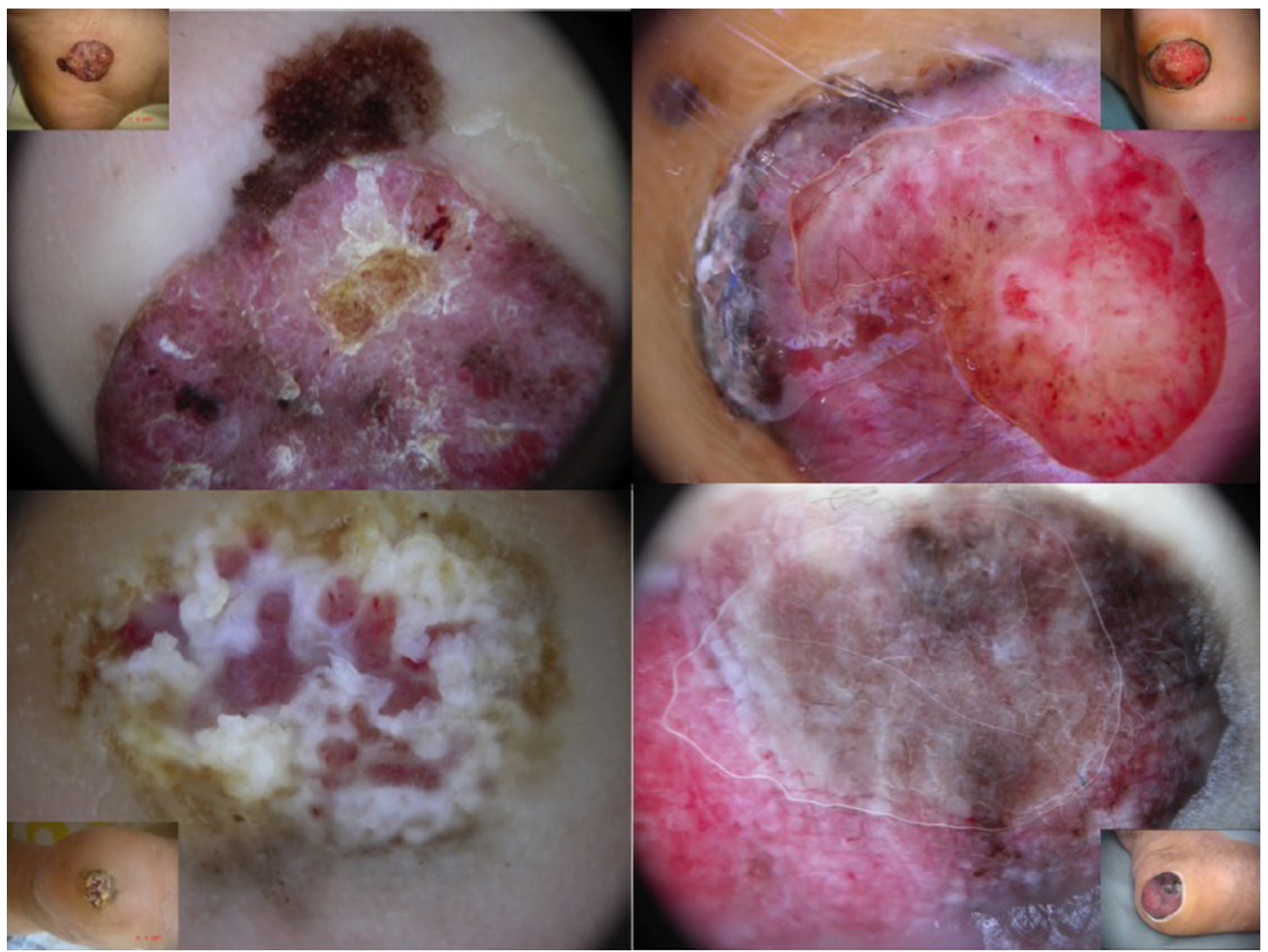

Figure 6. Examples of amelanotic melanomas showing remnants of pigmentation and atypical vessels on dermoscopy. [Copyright: @2018 Ozdemir et al.]

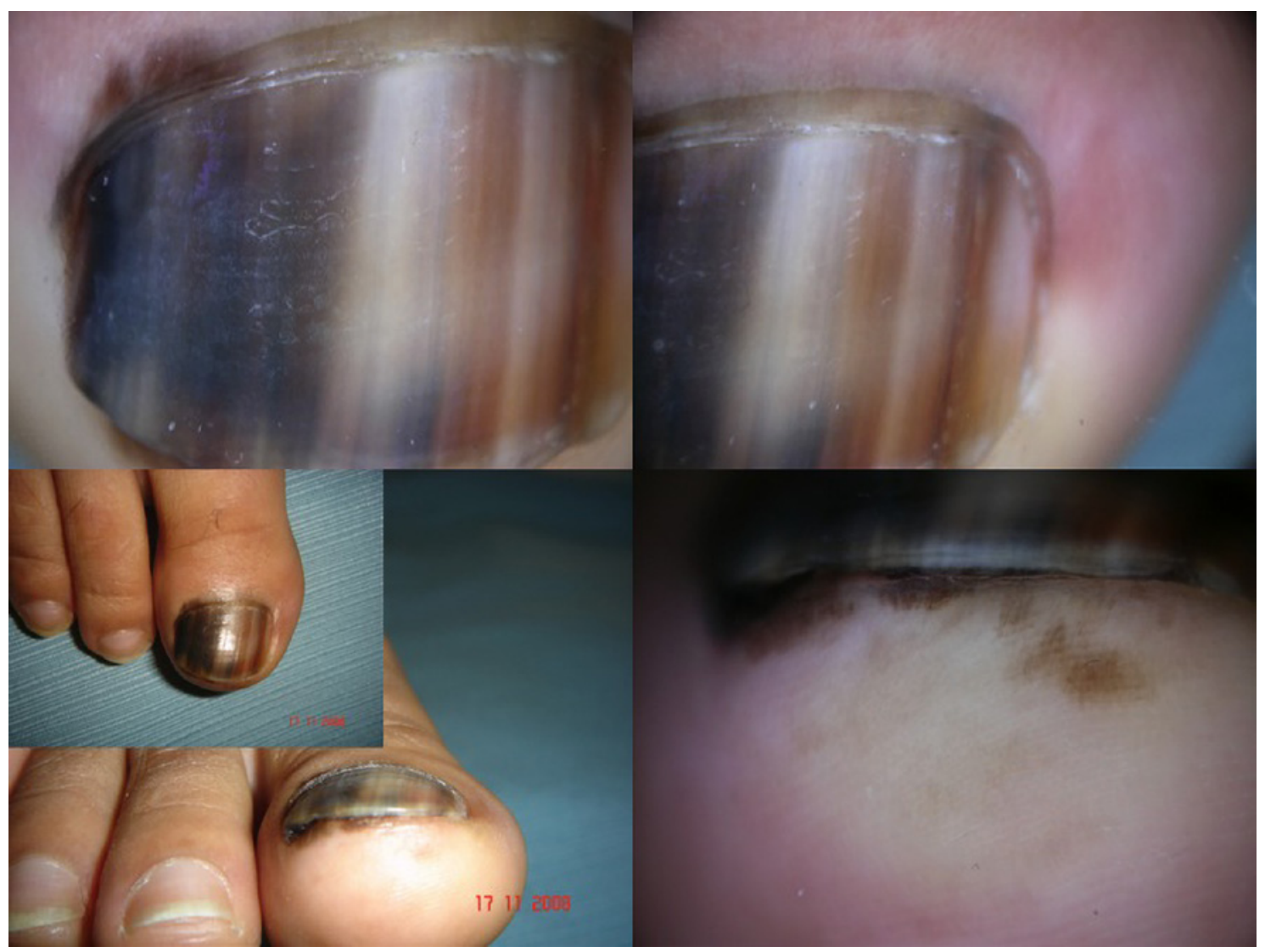

Figure 7. Nail unit melanoma dermoscopic features. Breslow $0.9 \mathrm{~mm}$, Clark III. The longitudinal bands show irregular colors and lack of parallelism. Hutchinson’s sign is detectable at clinical inspection. [Copyright: @2018 Ozdemir et al.] 


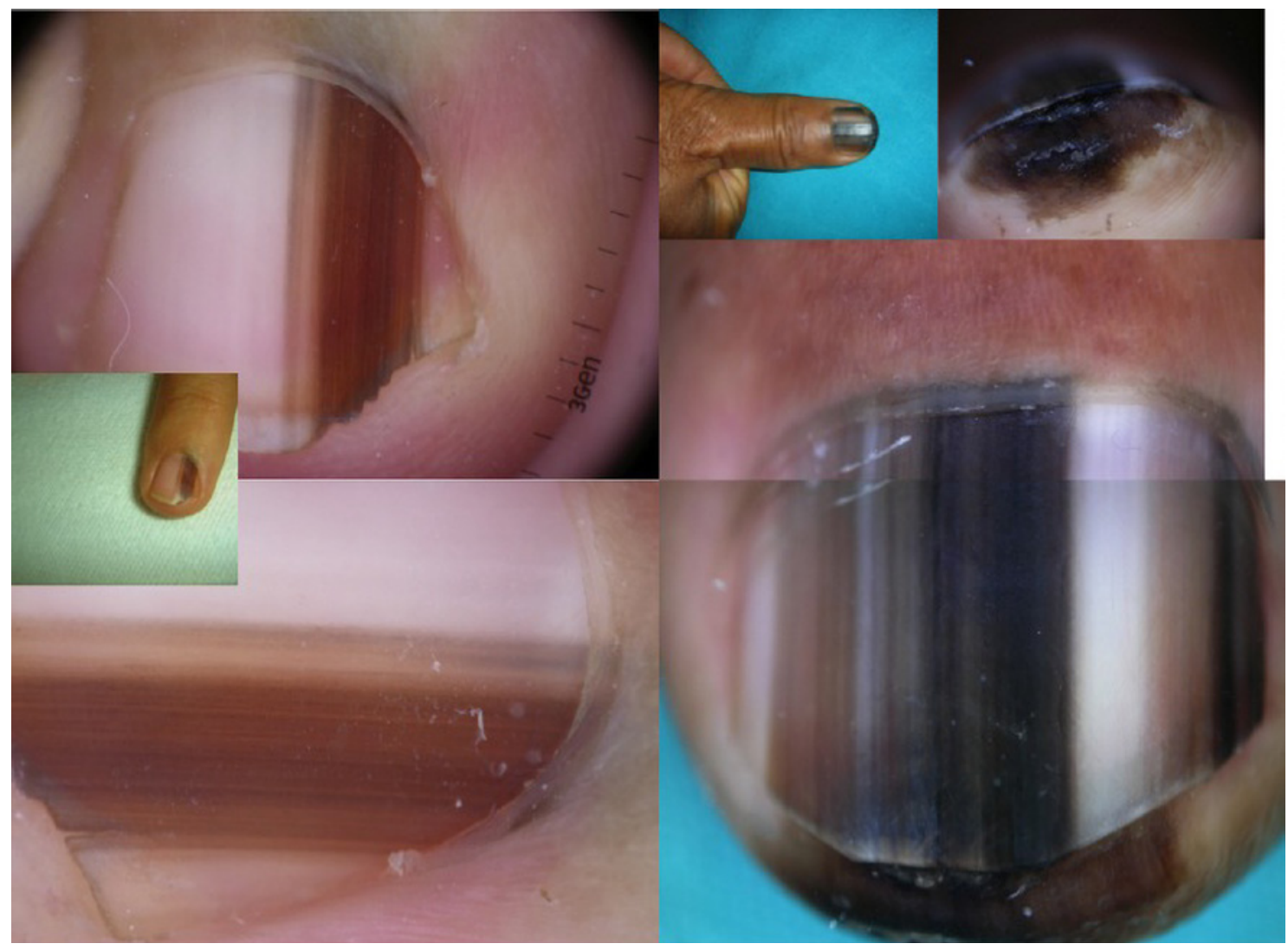

Figure 8. Nail unit melanoma dermoscopic features. Two in situ melanomas. The lines are irregular in thickness and spacing. [Copyright: @2018 Ozdemir et al.]

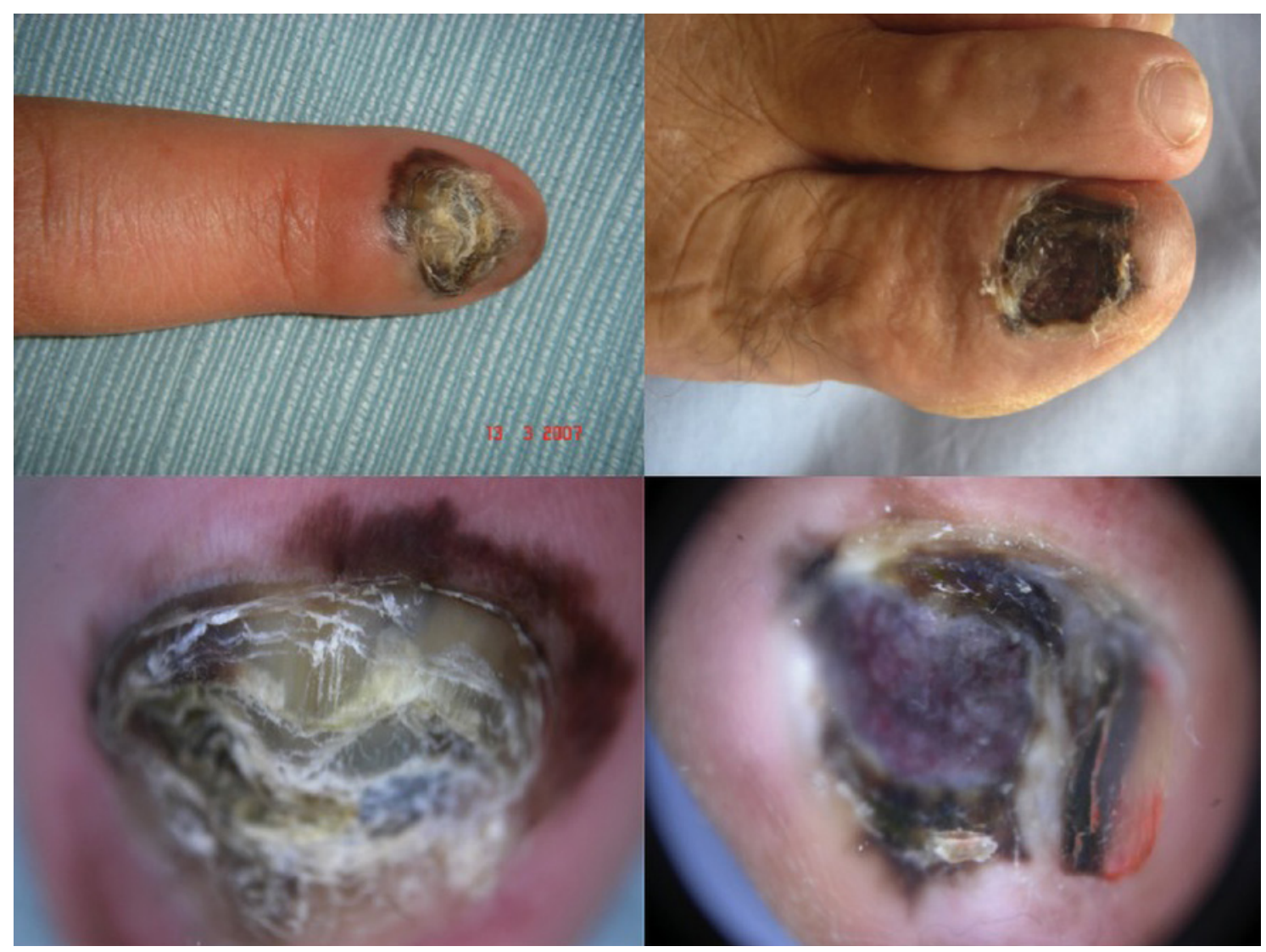

Figure 9. Nail unit melanoma dermoscopic features. (On the left) Breslow $2.7 \mathrm{~mm}$, Clark III. (On the right) Breslow $3.2 \mathrm{~mm}$, Clark IV. Both show nail plate dystrophy. Triangular shape of the band and microhemorrhage are detectable on the right. [Copyright: (2018 Ozdemir et al.] 
TABLE 2. Dermoscopic patterns of nail unit melanomas.

\begin{tabular}{|l|c|c|}
\multicolumn{1}{|c|}{$\begin{array}{c}\text { Dermoscopic } \\
\text { Patterns }\end{array}$} & $\begin{array}{c}\text { Number of } \\
\text { Subungual } \\
\text { Melanomas } \\
\text { (n, total = 11) }\end{array}$ & $\begin{array}{c}\text { Percentage } \\
\text { of Subungual } \\
\text { Melanomas } \\
\text { (\%) }\end{array}$ \\
\hline $\begin{array}{l}\text { Multicolored } \\
\text { background }\end{array}$ & 8 & 72.7 \\
\hline Brown background & 3 & 27.3 \\
\hline Irregular lines & 9 & 81.8 \\
\hline Blood spots & 6 & 54.5 \\
\hline $\begin{array}{l}\text { Linear } \\
\text { microhemorrhages }\end{array}$ & 2 & 18.2 \\
\hline $\begin{array}{l}\text { Triangular shape of the } \\
\text { band }\end{array}$ & 1 & 9 \\
\hline $\begin{array}{l}\text { Micro-Hutchinson's } \\
\text { sign }\end{array}$ & 0 & 0 \\
\hline
\end{tabular}

melanomas [35]. Steglich et al stated that melanoma initially shows dotted vessels and that with greater thickness, vascular polymorphism increases, with hairpin and linear-irregular vessels associated with milky-red areas. Dotted vessels may be the only clue to suspect non-pigmented melanoma [36]. Similar to thin amelanotic melanomas on non-glabrous skin showing only dotted vessels under dermoscopy, VPRP may be an important alerting sign for early diagnosis of ALM.

Melanoma cells, mostly among in situ melanomas, tend to cluster around the crista intermedia, leading to pigmentation on the ridges, seen as PRP on dermoscopy [22]. This may be due to the tendency, shown by melanocytic stem cells generating melanoma, to reside, at the beginning, near the crista intermedia or the eccrine ducts. On the other hand, in deeply invasive melanomas, tumor cells tend to diffusely proliferate with a similar intensity in both crista intermedia and crista limitans [22]. Since vascularization and inflammation is likely to be greater where the neoplastic melanocytes proliferate, we reason that in amelanotic cases, the increased vasculature will be most prominent along the same region, namely, the crista intermedia (Figure 10). This will manifest under dermoscopy as erythema and dotted vessels on the ridges, along the crista intermedia areas, accounting for the dermoscopic feature described herein as VPRP (Figure 11). In pigmented ALM, melanin obscures the increased vascularization leading to the PRP pattern. This is best seen in hypopigmented melanomas, whereby PRP in the lightly pigmented and VPRP in the amelanotic areas can both be seen.

\section{Conclusion}

In conclusion, we found that ALMs in Turkish patients display similar dermoscopic patterns as reported in other popu-

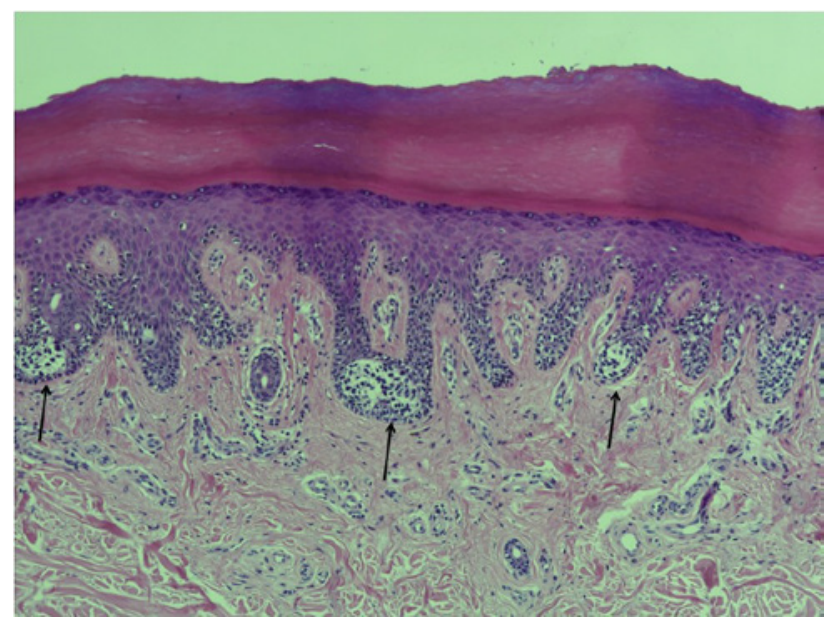

Figure 10. Histopathologic section of periphery of acral melanoma shown in Figure 2. Breslow $0.8 \mathrm{~mm}$, Clark II. Proliferation of nonpigmented cells may be identified around crista intermedia (arrows). Hematoxylin and eosin (H\&E) staining (original magnification x100). [Copyright: @2018 Ozdemir et al.]

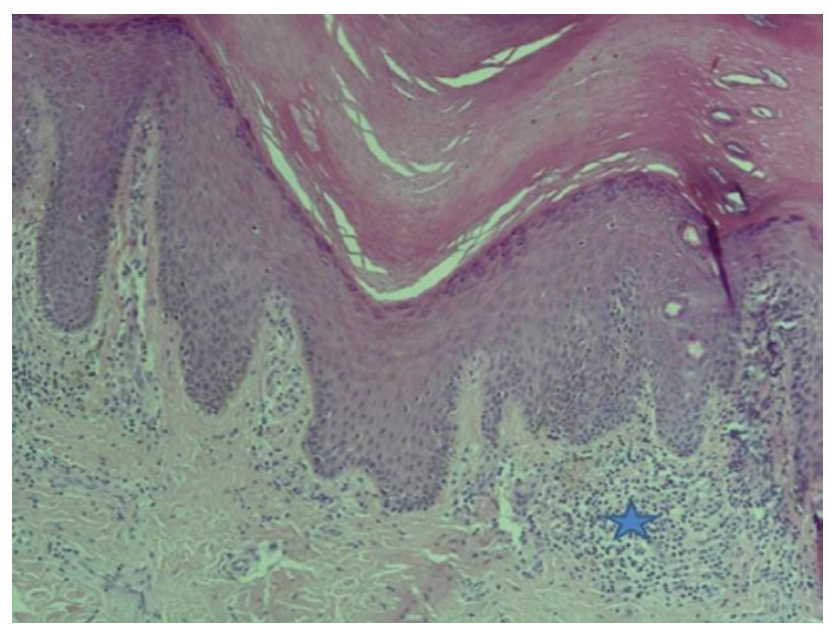

Figure 11. Histopathological section of periphery of acral melanoma shown in Figure 5. Breslow $2.26 \mathrm{~mm}$, Clark IV. Prominent vascular proliferation (asterisk) is detectable around crista intermedia/eccrine ducts, compared to other parts. H\&E staining (original magnification x100). [Copyright: @2018 Ozdemir et al.]

lations, with PRP being the most frequent. A lower incidence of IDP pattern may be related to the higher mean thickness of melanomas in our series. A newly described dermoscopic pattern, VPRP, may be important for the diagnosis of thin amelanotic ALMs. However, the significance of this dermoscopic feature needs to be validated in larger series.

\section{References}

1. Menzies SW, Kreusch J, Byth K, et al. Dermoscopic evaluation of amelanotic and hypomelanotic melanoma. Arch Dermatol. 2008;144(9):1120-127.

2. de Giorgi V, Sestini S, Massi D, Maio V, Giannotti B. Dermoscopy for "true" amelanotic melanoma: A clinical dermoscopy-pathologic case study. J Am Acad Dermatol. 2006;54(2):341-344. 
3. Moloney FJ, Menzies SW. Key points in the dermoscopic diagnosis of hypomelanotic melanoma and nodular melanoma. J Am Acad Dermatol. 2011;38(1):10-15.

4. Duarte AF, Correia O, Barros AM, Azevedo R, Haneke E. Nail matrix melanoma in situ: conservative surgical management. Dermatology. 2010;220(2):173-175.

5. Haneke E. Ungual melanoma-controversies in diagnosis and treatment. Dermatol Ther. 2012;25(6):510-524.

6. Chang JW. Acral melanoma: a unique disease in Asia. JAMA Dermatol. 2013;149(11):1272-1273.

7. Jung HJ, Kweon SS, Lee JB, Lee SC, Yun SJ. A clinicopathologic analysis of 177 acral melanomas in koreans: relevance of spreading pattern and physical stress. JAMA Dermatol. 2013;149(11):1281-1288.

8. Chang JW, Yeh KY, Wang $\mathrm{CH}$, et al. Malignant melanoma in Taiwan: a prognostic study of 181 cases. Melanoma Res. 2004;14(6):537-541.

9. Paladugu RR, Winberg CD, Yonemoto RH. Acral lentiginous melanoma. A clinicopathologic study of 36 patients. Cancer 1983;52(1):161-168.

10. O'Leary JA, Berend KR, Johnson JL, Levin LS, Seigler HF. Subungual melanoma. A review of 93 cases with identification of prognostic variables. Clin Orthop Relat Res. 2000;(378):206-212.

11. Banfield CC, Redburn JC, Dawber RP. The incidence and prognosis of nail apparatus melanoma. A retrospective study of 105 patients in four English regions. Br J Dermatol. 1998;139(2):276279

12. Rigby HS, Briggs JC. Subungual melanoma: a clinico-pathological study of 24 cases. Br J Plast Surg. 1992; 45(4):275-278.

13. Kato T, Suetake T, Sugiyama Y, Tabata N, Tagami H. Epidemiology and prognosis of subungual melanoma in 34 Japanese patients. Br J Dermatol. 1996;134(3):383-387.

14. Miura S, Jimbow K. Clinical characteristics of subungual melanomas in Japan: case report and a questionnaire survey of 108 cases. J Dermatol. 1985;12(5)393-402.

15. Phan A, Touzet S, Dalle S, Ronger-Savle' S, Balme B, Thomas L. Acral lentiginous melanoma: a clinicoprognostic study of 126 cases. Br J Dermatol. 2006;155(3):561-569.

16. Cascinelli N, Zurrida S, Galimberti V, et al. Acral lentiginous melanoma. A histological type without prognostic significance. J Dermatol Surg Oncol. 1994;20(12):817-822.

17. Kuchelmeister C, Schaumburg-Lever G, Garbe C. Acral cutaneous melanoma in Caucasians: clinical features, histopathology and prognosis in 112 patients. Br J Dermatol. 2000;143(2):275-280.

18. Slingluff CL Jr, Vollmer R, Seigler HF. Acral melanoma: a review of 185 patients with identification of prognostic variables. J Surg Oncol. 1990;45(2):91-98.

19. Saida T, Koga H, Uhara H. Key points in dermoscopic differentiation between early acral melanoma and acral nevus. J Dermatol. 2011;38(1):25-34.
20. Koga H, Saida T. Revised 3-step dermoscopic algorithm for the management of acral melanocytic lesions. Arch Dermatol. 2011;147(6):741-743.

21. Oguchi S, Saida T, Koganehira Y, Ohkubo S, Ishihara Y, Kawachi S. Characteristic epiluminescent microscopic features of early malignant melanoma on glabrous skin. A videomicroscopic analysis. Arch Dermatol. 1998;134(5):563-568.

22. Saida T, Oguchi S, Miyazaki A. Dermoscopy for acral pigmented lesions. Clin Dermatol. 2002;20(3):279-285.

23. Saida T, Miyazaki A, Oguchi S, et al. Significance of dermoscopic patterns in detecting malignant melanoma on acral volar skin: results of a multicenter study in Japan. Arch Dermatol. 2004;140(10):1233-1238.

24. Saida T. Lessons learned from studies of the development of early melanoma. Int J Clin Oncol. 2005;10(6):371-4.

25. Saida T, Oguchi S, Ishihara Y. In vivo observation of magnified features of pigmented lesions on volar skin using video macroscope. Arch Dermatol. 1995;131(3):298-304.

26. Malvehy J, Puig S. Dermoscopic patterns of benign volar melanocytic lesions in patients with atypical mole syndrome. Arch Dermatol. 2004;140(5):538-544.

27. Ozdemir F, Karaarslan IK, Akalin T. Variations in the dermoscopic features of acquired acral melanocytic nevi. Arch Dermatol. 2007;143(11):1378-1384.

28. Saida T, Koga H. Dermoscopic patterns of acral melanocytic nevi: their variations, changes, and significance. Arch Dermatol. 2007;143(11):1423-1426.

29. Lallas A, Sgouros D, Zalaudek I, et al. Palmar and plantar melanomas differ for sex prevalence and tumor thickness but not for dermoscopic patterns. Melanoma Res. 2014;24(1):83-87.

30. Braun RP, Thomas L, Dusza SW, et al. Dermoscopy of acral melanoma: a multicenter study on behalf of the International Dermoscopy Society. Dermatology. 2013;227(4):373-380.

31. Phan A, Dalle S, Touzet S, Ronger-Savlé S, Balme B, Thomas L. Dermoscopic features of acral lentiginous melanoma in a large series of 110 cases in a white population. Br J Dermatol. 2010; 162(4):765-771.

32. Argenziano G, Soyer HP, Chimenti S, et al. Dermoscopy of pigmented skin lesions: results of a consensus meeting via the Internet. J Am Acad Dermatol. 2003;48(5):679-693.

33. Argenziano G, Catricalà C, Ardigo M, et al. Seven-point checklist revisited. Br J Dermatol. 2011;164(4):785-790.

34. Kato T, Tabata N, Suetake T, Tagami H. Non-pigmented nodular plantar melanoma in 12 Japanese patients. Br J Dermatol. 1997;136(2):207-211.

35. Argenziano G, Zalaudek I, Corona R, et al. Vascular structures in skin tumors: a dermoscopy study. Arch Dermatol. 2004;140(12):1485-1489.

36. Steglich RB, Meotti CD, Ferreira MS, Lovatto L, de Carvalho AV, de Castro CG. Dermoscopic clues in the diagnosis of amelanotic and hypomelanotic malignant melanoma. An Bras Dermatol. 2012;87(6):920-923. 